\title{
Vascular structures for volumetric cooling and mechanical strength
}

\author{
K.-M. Wang, ${ }^{1}$ S. Lorente ${ }^{2}$ and A. Bejan ${ }^{3, a)}$ \\ ${ }^{1}$ Department of Mechanical Engineering, Tatung University, Taipei 104, Taiwan \\ ${ }^{2}$ LMDC (Laboratoire Matériaux et Durabilité des Constructions), INSA, Université de Toulouse, 135, \\ avenue de Rangueil, F-31 077 Toulouse Cedex 04, France \\ ${ }^{3}$ Department of Mechanical Engineering and Materials Science, Duke University, Durham, North Carolina \\ 27708-0300, USA
}

(Received 17 November 2009; accepted 17 December 2009; published online 17 February 2010)

\begin{abstract}
When solid material is removed in order to create flow channels in a load carrying structure, the strength of the structure decreases. On the other hand, a structure with channels is lighter and easier to transport as part of a vehicle. Here, we show that this trade off can be used for benefit, to design a vascular mechanical structure. When the total amount of solid is fixed and the sizes, shapes, and positions of the channels can vary, it is possible to morph the flow architecture such that it endows the mechanical structure with maximum strength. The result is a multifunctional structure that offers not only mechanical strength but also new capabilities necessary for volumetric functionalities such as self-healing and self-cooling. We illustrate the generation of such designs for strength and fluid flow for several classes of vasculatures: parallel channels, trees with one, two, and three bifurcation levels. The flow regime in every channel is laminar and fully developed. In each case, we found that it is possible to select not only the channel dimensions but also their positions such that the entire structure offers more strength and less flow resistance when the total volume (or weight) and the total channel volume are fixed. We show that the minimized peak stress is smaller when the channel volume $(\phi)$ is smaller and the vasculature is more complex, i.e., with more levels of bifurcation. Diminishing returns are reached in both directions, decreasing $\phi$ and increasing complexity. For example, when $\phi=0.02$ the minimized peak stress of a design with one bifurcation level is only $0.2 \%$ greater than the peak stress in the optimized vascular design with two levels of bifurcation.

(C) 2010 American Institute of Physics. [doi:10.1063/1.3294697]
\end{abstract}

\section{INTRODUCTION}

The thermal management of future air vehicles will require an integrative design of cooling, heating and mechanical strength functionalities throughout the aircraft structure. For example, the traditional approach to thermal management is as a self-standing and local capability, an add-on separate from mechanical strength, electrical power distribution, and other capabilities. In current designs, cooling technology is highly localized, in the core of the engine. The cooling technology is devoted to the hot gas path, and it has reached its limits: to cool and protect the mechanical structure along the hot gas path requires more and more bleed air from the compressor. This has a classical and negative impact on the thermodynamic performance of the whole vehicle [e.g., Ref. 1, pp. 418-420].

The cooling technology can be moved forward by liberating and morphing the architecture of the aircraft so that thermal and mechanical capabilities are designed together, and from the beginning. In this paper, we illustrate this principle, to conceptualize the aircraft structure as molding solid with flow spaces allocated to the subvolumes that need the fluid and heat currents that must flow. Mechanical structural members are components for the flow of stresses. ${ }^{2}$ Their proper sizes come from the same principle. Larger is better when designed alone (stresses are lower, materials cheaper,

a)Electronic mail: abejan@duke.edu. and manufacturing easier). Smaller is better when it is to be carried on board. The clash between the two trends determines the right sizes (weights, volumes). The weight and the volume of the component are two constraints that must be respected as we search for the multiobjective architectures proposed here. In this mass we distribute the finite-size flow volume so that the whole aircraft is more efficient. If the trade offs are in terms of destroyed useful energy (fuel), then the proposed architectures assists the evolution of the aircraft toward its energy-based design.

Vehicles of the future will require highly reliable structural systems capable of withstanding prolonged and repeated exposures to extreme environments including high levels of thermal input and structural loads over large surfaces. Thermal protection is required for the long term (steady state), not on a one-shot basis. To that end, a method to provide distributed cooling and structural strength will be very beneficial.

Dendritic flow structures are the most common designs encountered in nature. They are also a frequent subject of self-organization and self-optimization study in physics and biology. More recently, tree networks became a signature of constructal theory, which introduced them in engineering. ${ }^{1,2}$ To design with constructal theory is to discover the flow configuration by following a principle of greater flow access globally, in a flow and mechanical structure that is free to morph during its design. This activity is showing repeatedly and convincingly that dendritic flow structures are superior 


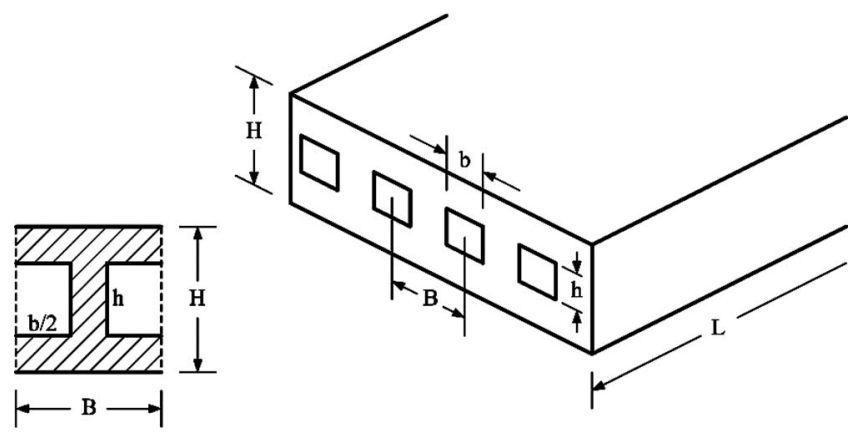

FIG. 1. Slab with equidistant parallel channels.

to conventional arrays of parallel channels for bathing entire areas and volumes. They are superior not only for maximizing fluid transport but also for maximizing volumetric heat and mass exchange. As in the blood flow on the underside of the ear of the elephant, dendritic flow is the best way to cool a stream with a surface (or to cool a surface with a stream).

At first sight, the embedding of tree channels in a structural member seems to be detrimental. The void spaces reserved for the flowing fluid could have been used for mechanical strength, by filling it with solid that can withstand high stresses. This view is correct as stated, however, here we propose to turn it inside out and exploit it for benefit. Key is the integrative approach that we take in the design of the entire aircraft: the objective is the best vehicle, not the perfect organ or component. When the total amount of solid is fixed and the channel sizes and positions can vary, it is possible to morph the architecture so that some of its currents are impeded-heat, fluid, and stresses. The result is a multifunctional structure that offers mechanical strength and volumetric functionalities such as self-cooling, self-healing, and volume averaged transport properties that can be made to vary according to need. ${ }^{3-19}$

\section{PARALLEL CHANNELS}

A simple illustration of how this trade-off leads to the channel configuration that preserves the mechanical strength properties of the wall is shown in Fig. 1. Consider a twodimensional slab with parallel channels separated by the distance B. The wall thickness is H. For simplicity assume that the channel cross-section is the rectangle $h \times b$.

Two objectives are in play, large mechanical strength and small flow resistance. The mechanical strength is characterized by the resistance modulus of the I-beam cross section $^{20}$

$$
\mathrm{W}=\frac{\mathrm{BH}^{3}-\mathrm{bh}^{3}}{6 \mathrm{H}} .
$$

The volume fraction occupied by the channels is

$$
\phi=\frac{\mathrm{bh}}{\mathrm{BH}} \text {. }
$$

For the flow resistance of a single channel of mass flow rate $\dot{\mathrm{m}}_{1}$ and length $\mathrm{L}$ we write

$$
\Delta \mathrm{P}=\mathrm{f} \frac{4 \mathrm{~L}}{\mathrm{D}_{\mathrm{h}}} \frac{1}{2} \rho \mathrm{U}^{2},
$$

where

$$
\begin{aligned}
& \mathrm{U}=\frac{\dot{\mathrm{m}}_{1}}{\rho \mathrm{hb}}, \\
& \mathrm{D}_{\mathrm{h}}=\frac{4 \mathrm{hb}}{2(\mathrm{~h}+\mathrm{b})}, \\
& \mathrm{f} \cong \frac{\mathrm{h}^{2}+\mathrm{b}^{2}}{(\mathrm{~h}+\mathrm{b})^{2}} \frac{24}{\operatorname{Re}_{\mathrm{D}_{\mathrm{h}}}}, \\
& \operatorname{Re}_{\mathrm{D}_{\mathrm{h}}}=\frac{\mathrm{UD} \mathrm{h}}{\nu} .
\end{aligned}
$$

Equation (6) is an adequate approximation for the friction factor for fully developed laminar flow through a rectangular channel (see Ref. 21, pp. 110). Combining Eqs. (3)-(7), we find

$$
\Delta \mathrm{P}=\left(24 \mathrm{~L} \nu \frac{\dot{\mathrm{m}}_{1}}{\mathrm{~B}}\right) \frac{\mathrm{B}\left(\mathrm{h}^{2}+\mathrm{b}^{2}\right)}{\mathrm{h}^{3} \mathrm{~b}^{3}} .
$$

The quantity inside the first set of brackets is a constant. During the bathing of a vascularized wall, the specified flow rate is the total flow rate in the $\mathrm{L}$ direction, not $\dot{\mathrm{m}}_{1}$. This means that the fixed quantity is the one-channel mass flow rate per unit width $\mathrm{B}$. The quantity that varies with the geometry is $\mathrm{B}\left(\mathrm{h}^{2}+\mathrm{b}^{2}\right) / \mathrm{h}^{3} \mathrm{~b}^{3}$, or the dimensionless function

$$
\mathrm{P}=\frac{\delta\left(\alpha^{2}+\beta^{2}\right)}{\alpha^{3} \beta^{3}},
$$

where

$$
\alpha=\frac{\mathrm{h}}{\mathrm{H}}, \quad \beta=\frac{\mathrm{b}}{\mathrm{H}}, \quad \delta=\frac{\mathrm{B}}{\mathrm{H}} .
$$

The corresponding dimensionless resistance modulus (stiffness) and void fraction [Eqs. (1) and (2)] are

$$
\begin{aligned}
& \mathrm{S}=\delta-\beta \alpha^{3}, \\
& \phi=\frac{\alpha \beta}{\delta} .
\end{aligned}
$$

The challenge is to find the geometry $(\alpha, \beta, \delta)$ that promises small $\mathrm{P}$ and small $1 / \mathrm{S}$ at the same time. To illustrate that such configurations exist, assume that $\mathrm{B}=\mathrm{H}$. This corresponds to $\mathrm{B}=\mathrm{d}$ and $\mathrm{H}=\mathrm{d}$, where $\mathrm{d}$ is the smallest length scale of the architecture. Therefore we set $\delta=1$ in Eqs. (9), (11), and (12), and after eliminating $\beta$ we obtain

$$
\begin{aligned}
& \mathrm{P}=\frac{\alpha^{2}}{\phi^{3}}+\frac{1}{\phi \alpha^{2}}, \\
& \frac{1}{\mathrm{~S}}=\frac{1}{1-\phi \alpha^{2}} .
\end{aligned}
$$



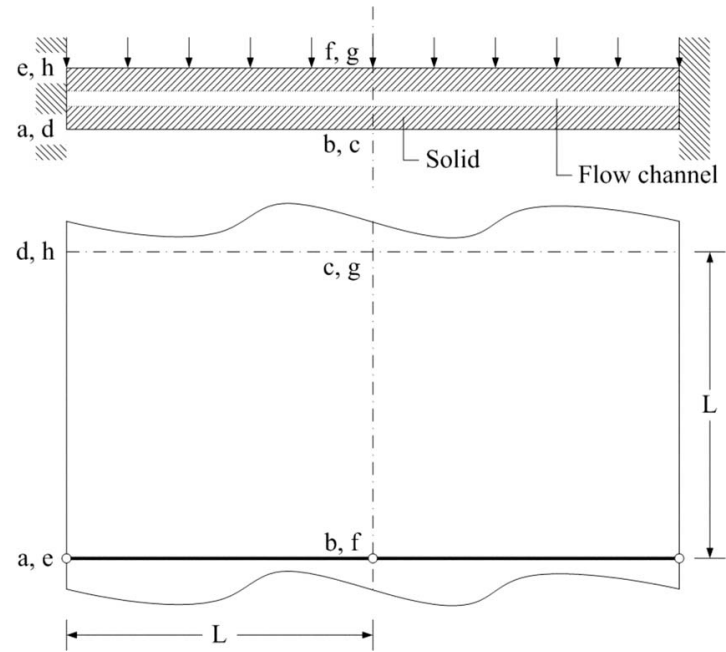

FIG. 2. Plate with implanted left and right edges, and uniformly distributed downward force.

Equations (13) and (14) establish $\mathrm{P}$ as a parametric function of $\mathrm{S}^{-1}$, where the parameter $\alpha$ covers the range $0<\alpha<1$. The minimum of each constant- $\phi$ curve occurs when the two terms in Eq. (13) are equal to each other. This means that the optimal channel height is

$$
\alpha=\phi^{1 / 2} \text {. }
$$

From Eq. (12) we find that the corresponding channel width is $\beta=\phi^{1 / 2}$. In conclusion, the $\mathrm{P}$ minima $\left(\mathrm{P}_{\min }=2 / \phi^{2}\right)$ correspond to square channel cross sections, not slender rectangles. Equation (14) becomes $S=1-\phi^{2}$. Eliminating $\phi$ between $\mathrm{P}=2 / \phi^{2}$ and $\mathrm{S}=1-\phi^{2}$, we conclude that the minima of the constant- $\phi$ curves lie on the curve

$$
P_{\min }=\frac{2 S^{-1}}{S^{-1}-1}=\frac{2}{1-S}
$$

The corresponding wall stiffness decreases monotonically as the minimized flow resistance decreases,

$$
\mathrm{S}=1-\frac{2}{\mathrm{P}_{\min }} .
$$

This indicates again the trade-off that exists between mechanical strength and fluid flow resistance.

\section{TREE-SHAPED CHANNELS}

Consider next the plate with embedded flow channels shown in Fig. 2. The top figure shows the profile of the plate. The plate is implanted in the two walls shown left and right. A uniformly distributed force is applied downward on the top surface. We are interested in morphing the flow structures to minimize the loss in mechanical strength due to the presence of channels.

The plate is composed of many elemental volumes of type abcdhgfe, which is shown in more detail in Fig. 3. This elemental volume is the domain selected for numerical simulation. The dimensions of this elemental volume are $\mathrm{L} \times \mathrm{L}$ $\times 0.1 \mathrm{~L}(\mathrm{ab} \times \mathrm{bc} \times \mathrm{cg})$. One end of the flow channel (the in-

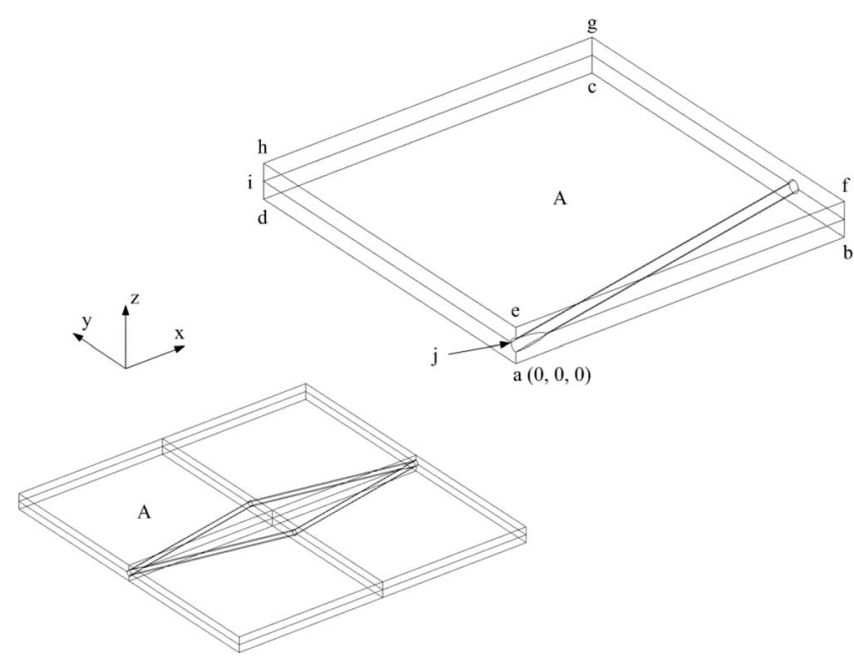

FIG. 3. Elemental volume of the plate shown in Fig. 2.

let) is in the middle of edge ae. The location of the other end (the outlet) is at $x=\mathrm{L}, z=0.05 \mathrm{~L}$ and $0 \leqq y \leqq \mathrm{~L}$. The following boundary conditions are specified:

- Surfaces ajid and jehi have zero displacement, $x=0$ and $y=0$ (no $x$ and $y$ displacement).

- The edge ij has fixed prescribed displacement, $z=0$ (no $z$ displacement).

- Surfaces abfe, bcgf, and cdhg are symmetry planes.

- Downward force F is applied uniformly on the surface efgh of the volume element.

The cross section of the channel embedded in this material is circular rather than rectangular as in Sec. II and Fig. 1. We chose circular cross sections in order to minimize stress concentrations. Yet, the lessons that we develop here with circular cross sections would be the same if we were to use rectangular cross sections.

The search is for the optimal location of the outlet so that the whole body has maximal stiffness. To achieve this, we simulated numerically the material displacement and stress fields throughout the volume occupied by solid. The conservation of momentum is expressed in terms of stresses,

$$
-\frac{\partial \sigma_{x}}{\partial x}-\frac{\partial \tau_{x y}}{\partial y}-\frac{\partial \tau_{x z}}{\partial z}=0,
$$

$$
\begin{aligned}
& -\frac{\partial \tau_{x y}}{\partial x}-\frac{\partial \sigma_{y}}{\partial y}-\frac{\partial \tau_{y z}}{\partial z}=0, \\
& -\frac{\partial \tau_{x z}}{\partial x}-\frac{\partial \tau_{y z}}{\partial y}-\frac{\partial \sigma_{z}}{\partial z}=0,
\end{aligned}
$$

where $\sigma$ and $\tau$ are the normal and shear stresses. The stressstrain relationship for an isotropic material is 


$$
\left[\begin{array}{c}
\varepsilon_{x} \\
\varepsilon_{y} \\
\varepsilon_{z} \\
\gamma_{x y} \\
\gamma_{y z} \\
\gamma_{x z}
\end{array}\right]=\frac{1}{\mathrm{E}}\left[\begin{array}{cccccc}
1 & -\nu & -\nu & 0 & 0 & 0 \\
-\nu & 1 & -\nu & 0 & 0 & 0 \\
-\nu & -\nu & 1 & 0 & 0 & 0 \\
0 & 0 & 0 & 2(1+\nu) & 0 & 0 \\
0 & 0 & 0 & 0 & 2(1+\nu) & 0 \\
0 & 0 & 0 & 0 & 0 & 2(1+\nu)
\end{array}\right]\left[\begin{array}{c}
\sigma_{x} \\
\sigma_{y} \\
\sigma_{z} \\
\tau_{x y} \\
\tau_{y z} \\
\tau_{x z}
\end{array}\right],
$$

where $\varepsilon$ is the normal strain, $\gamma$ is the shear strain, $\mathrm{E}$ is Young's modulus, and $\nu$ is Poisson's ratio. The straindisplacement relationships for small displacements are

$$
\begin{aligned}
& \varepsilon_{x}=\frac{\partial u}{\partial x}, \quad \varepsilon_{y}=\frac{\partial v}{\partial y}, \quad \varepsilon_{z}=\frac{\partial w}{\partial z}, \\
& \gamma_{x y}=\frac{\partial u}{\partial y}+\frac{\partial v}{\partial x}, \quad \gamma_{y z}=\frac{\partial v}{\partial z}+\frac{\partial w}{\partial y}, \quad \gamma_{x z}=\frac{\partial u}{\partial z}+\frac{\partial w}{\partial x},
\end{aligned}
$$

where $u, v$, and $w$ are the displacements in the $x, y$, and $z$ directions, respectively. The damping effect is assumed negligible. The governing equations were nondimensionalized by defining the dimensionless variables

$$
\begin{aligned}
& (\widetilde{u}, \widetilde{v}, \widetilde{w}, \widetilde{x}, \tilde{y}, \tilde{z})=(u, v, w, x, y, z) / \mathrm{L}, \\
& (\widetilde{\sigma}, \widetilde{\tau}, \widetilde{\mathrm{E}})=(\sigma, \tau, \mathrm{E}) \mathrm{L}^{2} / \mathrm{F} .
\end{aligned}
$$

Equations (18)-(23) become

$$
\begin{aligned}
& -\frac{\partial \widetilde{\sigma}_{x}}{\partial \widetilde{x}}-\frac{\partial \widetilde{\tau}_{x y}}{\partial \widetilde{y}}-\frac{\partial \widetilde{\tau}_{x z}}{\partial \widetilde{z}}=0, \\
& -\frac{\partial \widetilde{\tau}_{x y}}{\partial \widetilde{x}}-\frac{\partial \widetilde{\sigma}_{y}}{\partial \tilde{y}}-\frac{\partial \widetilde{\tau}_{y z}}{\partial \widetilde{z}}=0, \\
& -\frac{\partial \widetilde{\tau}_{x z}}{\partial \widetilde{x}}-\frac{\partial \widetilde{\tau}_{y z}}{\partial \tilde{y}}-\frac{\partial \widetilde{\sigma}_{z}}{\partial \widetilde{z}}=0,
\end{aligned}
$$

$$
\begin{aligned}
& \varepsilon_{x}=\frac{\partial \widetilde{u}}{\partial \widetilde{x}}, \quad \varepsilon_{y}=\frac{\partial \widetilde{v}}{\partial \widetilde{y}}, \quad \varepsilon_{z}=\frac{\partial \widetilde{w}}{\partial \widetilde{z}}, \\
& \gamma_{x y}=\frac{\partial \widetilde{u}}{\partial \widetilde{y}}+\frac{\partial \widetilde{v}}{\partial \widetilde{x}}, \quad \gamma_{y z}=\frac{\partial \widetilde{v}}{\partial \widetilde{z}}+\frac{\partial \widetilde{w}}{\partial \widetilde{y}}, \quad \gamma_{x z}=\frac{\partial \widetilde{u}}{\partial \widetilde{z}}+\frac{\partial \widetilde{w}}{\partial \widetilde{x}} .
\end{aligned}
$$

Numerical simulations were conducted in the dimensionless parametric domain represented by $\widetilde{\mathrm{E}}=2 \times 10^{5}, \nu=0.33$, an elemental volume of size $1 \times 1 \times 0.1$ and the uniformly distributed downward force 1 . For example, these dimensionless parameters correspond to $\mathrm{L}=1 \mathrm{~m}$ and $\mathrm{F} / \mathrm{L}^{2}$ $=10^{6} \mathrm{~Pa}$ when $\mathrm{E}=2 \times 10^{11} \mathrm{~Pa}$. The von Mises stress is used as the yield criterion,

$$
\widetilde{\sigma}_{\mathrm{vM}}=2^{-1 / 2}\left[\left(\widetilde{\sigma}_{x}-\widetilde{\sigma}_{y}\right)^{2}+\left(\widetilde{\sigma}_{y}-\widetilde{\sigma}_{z}\right)^{2}+\left(\widetilde{\sigma}_{z}-\widetilde{\sigma}_{x}\right)^{2}\right]^{1 / 2},
$$

and the objective of the search is to find configurations with smaller and smaller $\widetilde{\sigma}_{\mathrm{vM}}$. The computations were performed using a finite-element package ${ }^{22}$ with geometric multigrid linear system solver, SOR presmoother, SORU postsmoother, and UMFPACK for coarse solver. In order to verify that the solution is independent of grid size, the degrees of freedom (DOF, which increase as the number of elements increases) solved by the solver were increased in steps of $20 \%$ until the changes in maximum $z$-displacements and maximum von Mises stresses on the plane bcgf were within $1 \%$. The adaptive mesh refinement provided by COMSOL was utilized to improve the mesh refinement efficiency. The rough global minimum method was selected for the element selection method. The required mesh numbers varied from case to case; however, the minimum number of DOF solved by the solver was 76656.

\section{MECHANICAL STRENGTH}

Consider first the single channel flow structure shown in Fig. 3. The $y$ location of the outlet can vary. The porosity $\phi=\mathrm{V}_{\text {channel }} / \mathrm{V}_{\text {total }}$ is a fixed parameter, therefore the channel 

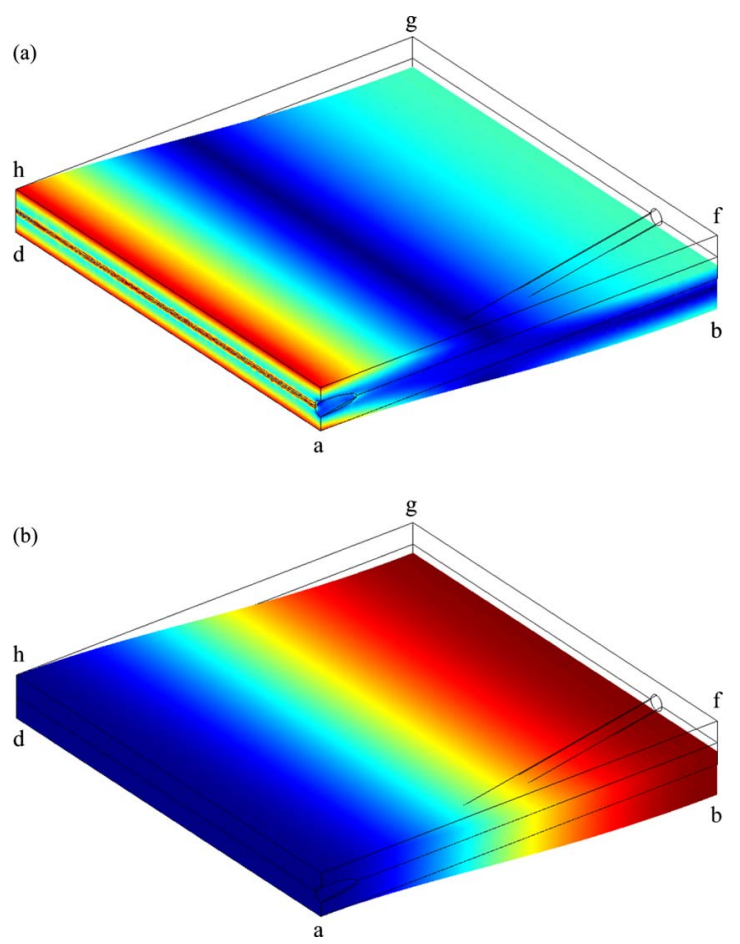

FIG. 4. (Color online) (a) Stress distribution in the deformed plate, and (b) $z$-displacement distribution $(y=0.2)$.

thicknesses will vary as the outlet $y$ location changes. Figure 4(a) shows the color contour plot of the von Mises stress on the deformed shape volume element when the is outlet at $y$ $=0.2$. The edges eh, ji, and ad are regions of high stresses, and the edge $\mathrm{ji}$ is suffering from the singularity problem indicating that the more meshes we concentrate on the edge $\mathrm{ji}$, the higher the stress we will calculate. According to the Saint Venant's principle, the singularity has no effect on the stress that is far away from the immediate region where the singularity occurs. Therefore we focus on the second high stress area, the plane bcgf. The objective is to find the optimal $y$ location of the outlet so that the maximum stress in plane bcgf is minimal. In Fig. 4(b) we show the color distribution of $z$ displacement when the outlet is at $y=0.2$.

Six outlet locations are considered, $y=0,0.2,0.4,0.6$, 0.8 , and 1 . Figure 5 shows the maximum von Mises stress in the plane bcgf for five different porosities, $\phi=0.01,0.02$, $0.03,0.05$, and 0.07 . The results show that the $y=0.2$ case provides the lowest maximum stress, that is, the highest mechanical strength, when $\phi=0.01,0.02,0.03$, and 0.05 . With $\phi=0.07$, the $y=1$ case is the best design. The worst case belongs to the $y=1$ design with $\phi=0.01$. In the case of $\phi$ $=0.02$ and 0.03 , the $y=0$ position is the worst. For $\phi=0.05$ and 0.07 , the worst cases are $y=0.8$ and $y=0.2$, respectively.

Important is to discover not only the best design but also the reduction in the maximum stress. The best configurations offer reductions of $1.3 \%, 6.3 \%, 53.8 \%, 19.4 \%$, and $29.6 \%$ in the maximum stresses (relative to the worst designs) when $\phi=0.01,0.02,0.03,0.05$, and 0.07 , respectively. This shows again the importance of morphing the flow architecture while placing more materials where material is needed.
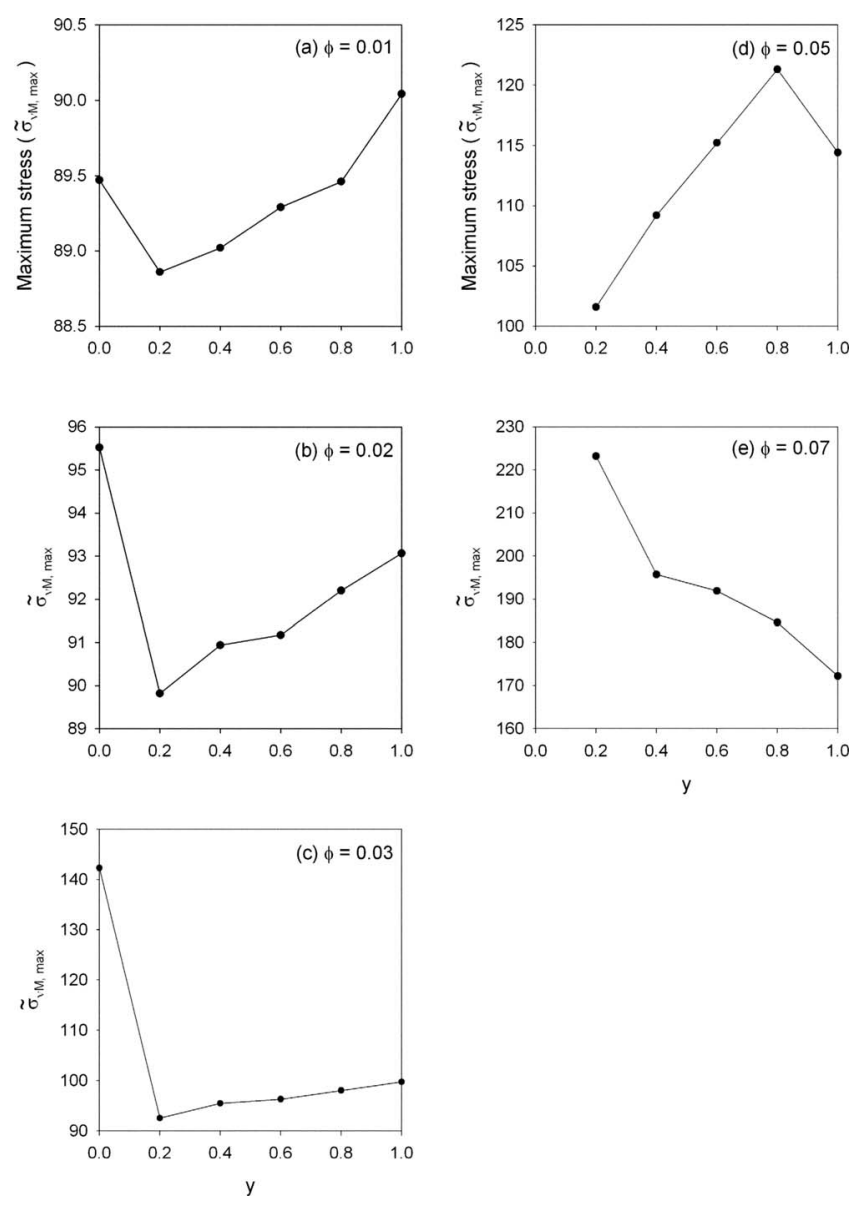

FIG. 5. Maximum stress in the plane bcgf of all the designs when $\phi$ $=0.01,0.02$, and 0.03 .

\section{ONE BIFURCATION LEVEL}

Next, we extended the search for maximum-strength structures by considering more complex flow configurations. In Fig. 6 the structure has one bifurcation, and there are three channel sizes, $\mathrm{L}_{1}, \mathrm{D}_{1}, \mathrm{~L}_{2}, \mathrm{D}_{2}, \mathrm{~L}_{3}$, and $\mathrm{D}_{3}$. Preliminary work on this configuration (Ref. 2, Problem 4.4) showed that the $y$-shaped flow structure has minimal flow resistance at fixed flow volume when its dimensions obey the proportions

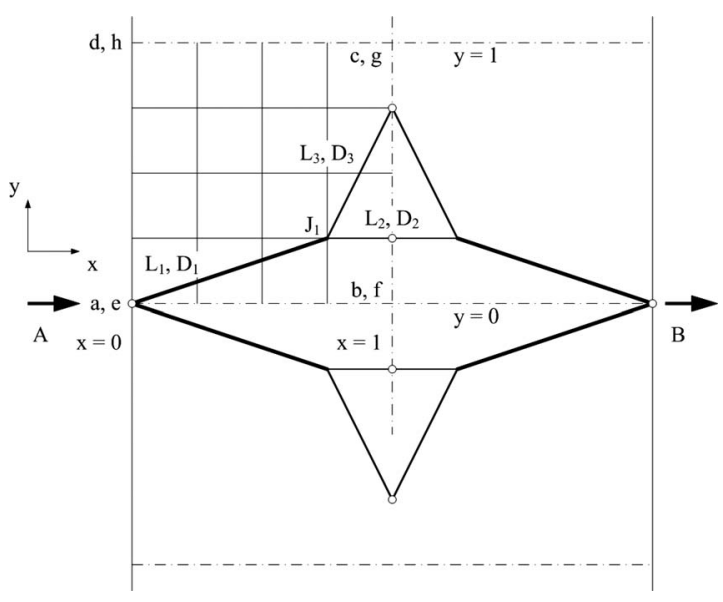

FIG. 6. Plate with channels with one bifurcation level. 

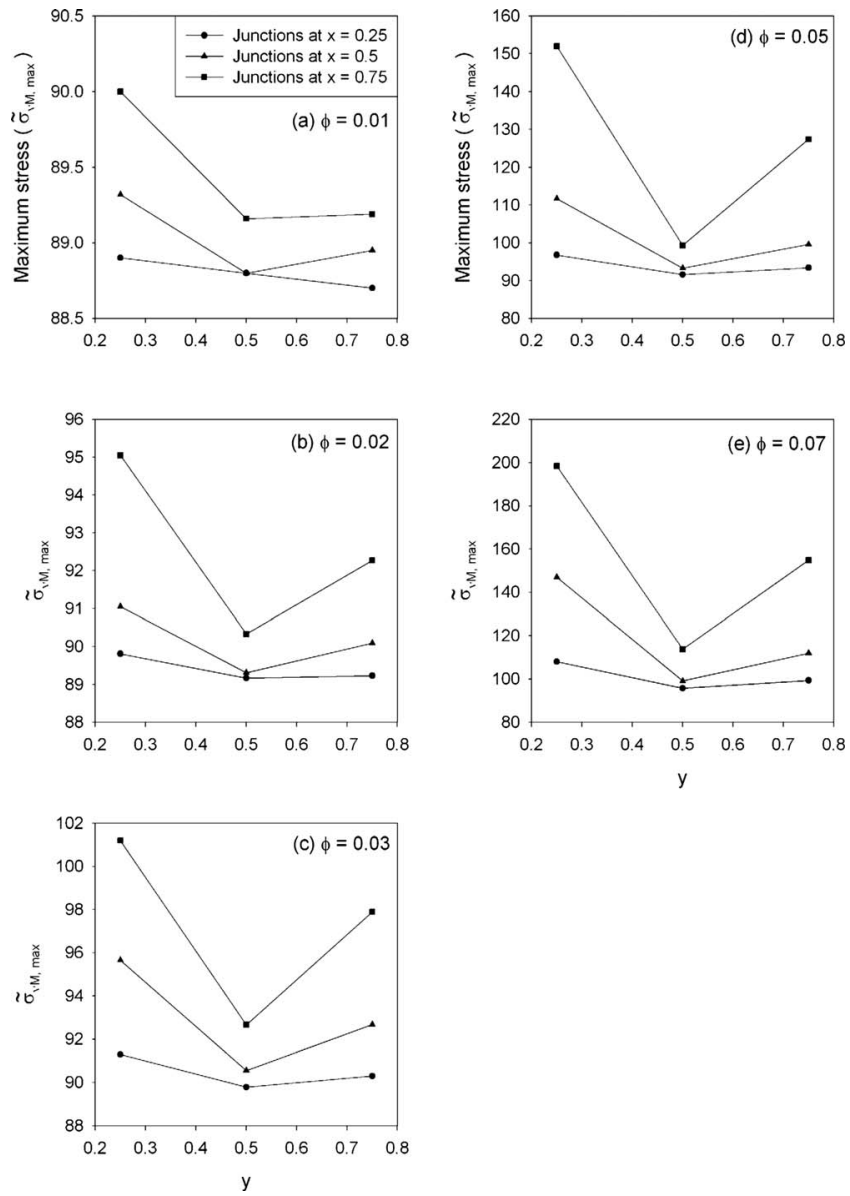

FIG. 7. Variation in the maximum stress with the location of junction $J_{1}$.

$$
\begin{aligned}
& \frac{\mathrm{D}_{1}}{\mathrm{D}_{2}}=\left[1+\left(\frac{\mathrm{L}_{3}}{\mathrm{~L}_{2}}\right)^{3}\right]^{1 / 3}, \\
& \frac{\mathrm{D}_{2}}{\mathrm{D}_{3}}=\frac{\mathrm{L}_{2}}{\mathrm{~L}_{3}}
\end{aligned}
$$

The locations of the outlets are fixed at $(x, y)=(1,0.25)$ and $(1,0.75)$. Free to change is the location of the junction $\left(J_{1}\right)$. To start the search for the design with the lowest stress, we divide the elemental volume into 16 square blocks. Each block has the size $0.25 \times 0.25 \times 0.1$. Nine locations of $\mathrm{J}_{1}$ are studied, their coordinates being a combinations of $x=0.25$, 0.5 , and 0.75 , and $y=0.25,0.5$, and 0.75 .

The maximum stresses reported in Fig. 7 reveal that $\mathrm{J}_{1}$ at $(x, y)=(0.75,0.25)$ is the worst. This design is the one illustrated in Fig. 6. The best location of $\mathrm{J}_{1}$ is at $(x, y)$ $=(0.25,0.75)$ when $\phi=0.01$, and it migrates to $(x, y)$ $=(0.25,0.5)$ when the porosity increases to 0.03 . The advantage of using the best configuration is a $1.5 \%, 6.6 \%, 12.7 \%$, $66 \%$, and $106 \%$ reduction in the maximum stress relative to the worst cases, as the porosity changes from 0.01, 0.02, $0.03,0.05$, and finally 0.07 .

\section{TWO BIFURCATION LEVELS}

The features of the designs with two bifurcations are shown in Fig. 8. Four outlets are placed at $(x, y)$ $=(1,0.125),(1,0.375),(1,0.625)$, and $(1,0.875)$. The loca-

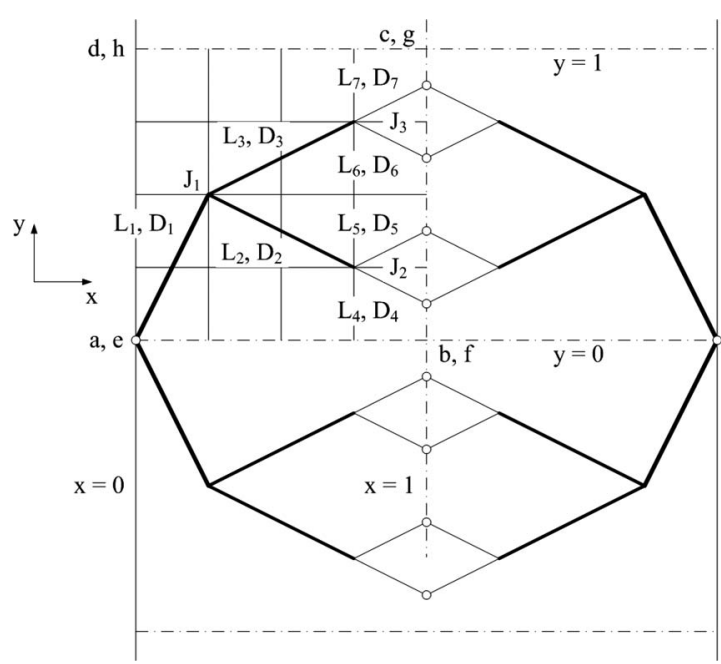

FIG. 8. Embedded tree-shaped flow structure with two bifurcation levels.

tions of junctions $\mathrm{J}_{2}$ and $\mathrm{J}_{3}$ are fixed at $(x, y)=(0.75,0.25)$ and $(0.75,0.75)$. The dimensions of channels $1-3$ are determined using Eqs. (33) and (34). The dimensions of channels 4-7 are based on the same principle, namely, minimal global flow resistance at fixed flow volume,

$$
\begin{aligned}
& \frac{\mathrm{D}_{2}}{\mathrm{D}_{4}}=\left[1+\left(\frac{\mathrm{L}_{5}}{\mathrm{~L}_{4}}\right)^{3}\right]^{1 / 3}, \quad \frac{\mathrm{D}_{4}}{\mathrm{D}_{5}}=\frac{\mathrm{L}_{4}}{\mathrm{~L}_{5}}, \\
& \frac{\mathrm{D}_{3}}{\mathrm{D}_{6}}=\left[1+\left(\frac{\mathrm{L}_{7}}{\mathrm{~L}_{6}}\right)^{3}\right]^{1 / 3}, \quad \frac{\mathrm{D}_{6}}{\mathrm{D}_{7}}=\frac{\mathrm{L}_{6}}{\mathrm{~L}_{7}} .
\end{aligned}
$$

We studied six locations for the junction $\mathrm{J}_{1}$, each being a combination of $x=0.25$ and 0.5 and $y=0.25,0.5$, and 0.75 , and searched for the location that offers the lowest maximum stress.

We show in Fig. 9 the maximum stresses in designs with $\phi=0.01,0.02,0.03,0.05$, and 0.07 . The $\mathrm{J}_{1}$ located at $(x, y)$ $=(0.5,0.25)$ yields the highest maximum stress among the six locations while the best location is at $(x, y)$ $=(0.25,0.75)$. When the porosity is less than 0.03 , the improvement in adopting the best location offers a stress reduction of less than $1 \%$. However, the trend confirms the need to adopt a designed configuration, as the best design of Fig. 9 (e) offers a $6.8 \%$ reduction in the maximum stress. The optimized configurations with one and two bifurcation levels are illustrated in Fig. 10 by showing the contour plot of the von Mises stress distribution on the elemental volumes with deformation.

\section{THREE BIFURCATION LEVELS}

The design with three bifurcations is shown in Fig. 11. We fixed $\mathrm{J}_{2}$ and $\mathrm{J}_{3}$ at $x=0.5, y=0.25$ and $0.75 ; \mathrm{J}_{4}, \mathrm{~J}_{5}, \mathrm{~J}_{6}$, and $\mathrm{J}_{7}$ at $x=0.75, y=0.125,0.375,0.625$, and 0.875 . The $x$ location of $\mathrm{J}_{1}$ was fixed at 0.25 , and the $y$ locations of $\mathrm{J}_{1}$ are 0.25 , 0.5 , and 0.75 . The dimensions of channels $1-7$ are the same as in Eqs. (33)-(36). The dimensions of channels 8-15 are such that 

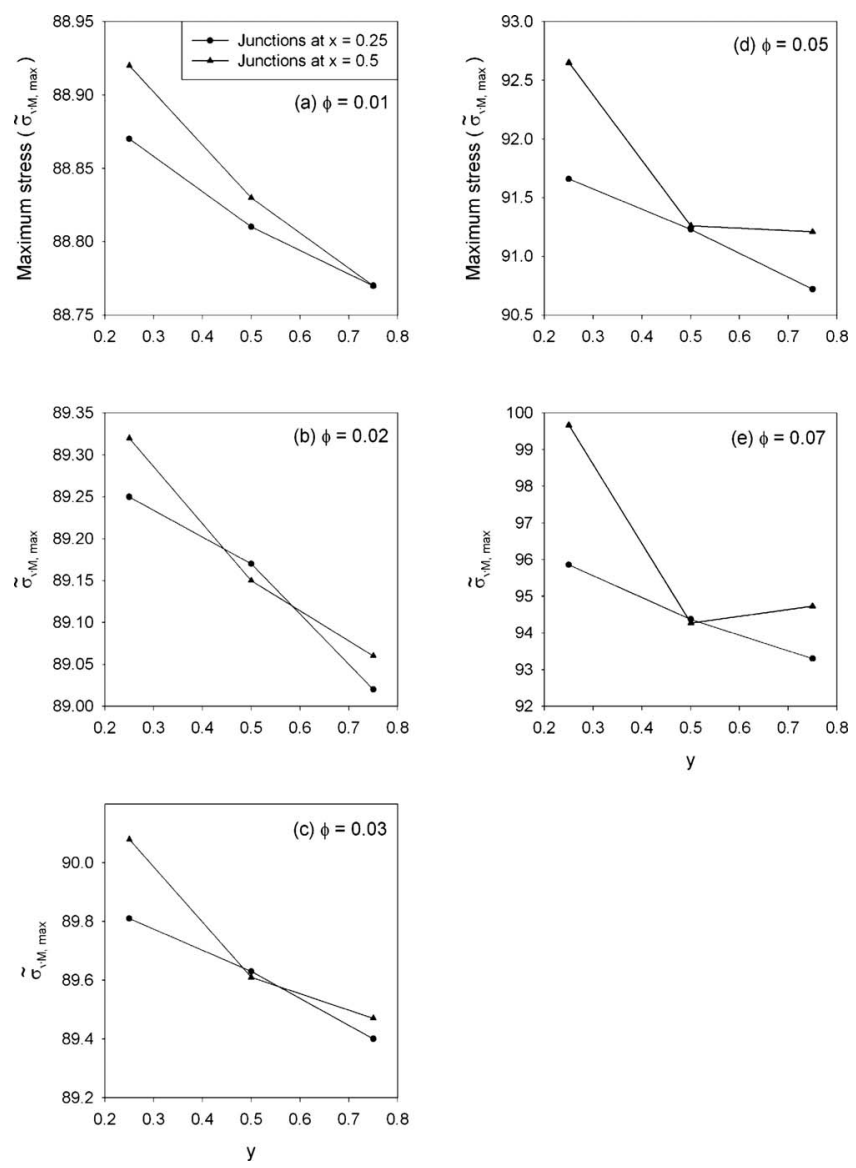

FIG. 9. Maximum stresses in the design of Fig. 8 when $\phi=0.01,0.02,0.03$, 0.05 , and 0.07 .

$$
\begin{aligned}
& \frac{\mathrm{D}_{4}}{\mathrm{D}_{8}}=\left[1+\left(\frac{\mathrm{L}_{9}}{\mathrm{~L}_{8}}\right)^{3}\right]^{1 / 3}, \quad \frac{\mathrm{D}_{8}}{\mathrm{D}_{9}}=\frac{\mathrm{L}_{8}}{\mathrm{~L}_{9}}, \\
& \frac{\mathrm{D}_{5}}{\mathrm{D}_{10}}=\left[1+\left(\frac{\mathrm{L}_{11}}{\mathrm{~L}_{10}}\right)^{3}\right]^{1 / 3}, \quad \frac{\mathrm{D}_{10}}{\mathrm{D}_{11}}=\frac{\mathrm{L}_{10}}{\mathrm{~L}_{11}}, \\
& \frac{\mathrm{D}_{6}}{\mathrm{D}_{12}}=\left[1+\left(\frac{\mathrm{L}_{13}}{\mathrm{~L}_{12}}\right)^{3}\right]^{1 / 3}, \quad \frac{\mathrm{D}_{12}}{\mathrm{D}_{13}}=\frac{\mathrm{L}_{12}}{\mathrm{~L}_{13}}, \\
& \frac{\mathrm{D}_{7}}{\mathrm{D}_{14}}=\left[1+\left(\frac{\mathrm{L}_{15}}{\mathrm{~L}_{14}}\right)^{3}\right]^{1 / 3}, \quad \frac{\mathrm{D}_{14}}{\mathrm{D}_{15}}=\frac{\mathrm{L}_{14}}{\mathrm{~L}_{15}} .
\end{aligned}
$$

We are interested in the $y$ location of $\mathrm{J}_{1}$ that provides the lowest maximum stress. Figure 12 shows that the best $y$ location is 0.75 and the worst one is 0.25 , and the improvement of adopting the best design is less than $1 \%$ in the reduction of maximum stress of the worst case.

In Fig. 13 we show that when the porosity is higher than 0.02 , the optimized tree-shaped configurations are helpful in reducing the maximum stresses. The higher the porosity, the more improvement in the maximum stress reduction we can expect from adopting designed tree-shaped configurations.

In Fig. 14 we summarize the results in terms of global fluid-flow performance. The flow along every channel is in the Poiseuille regime, and the junction losses are negligible. The global flow resistance is evaluated from
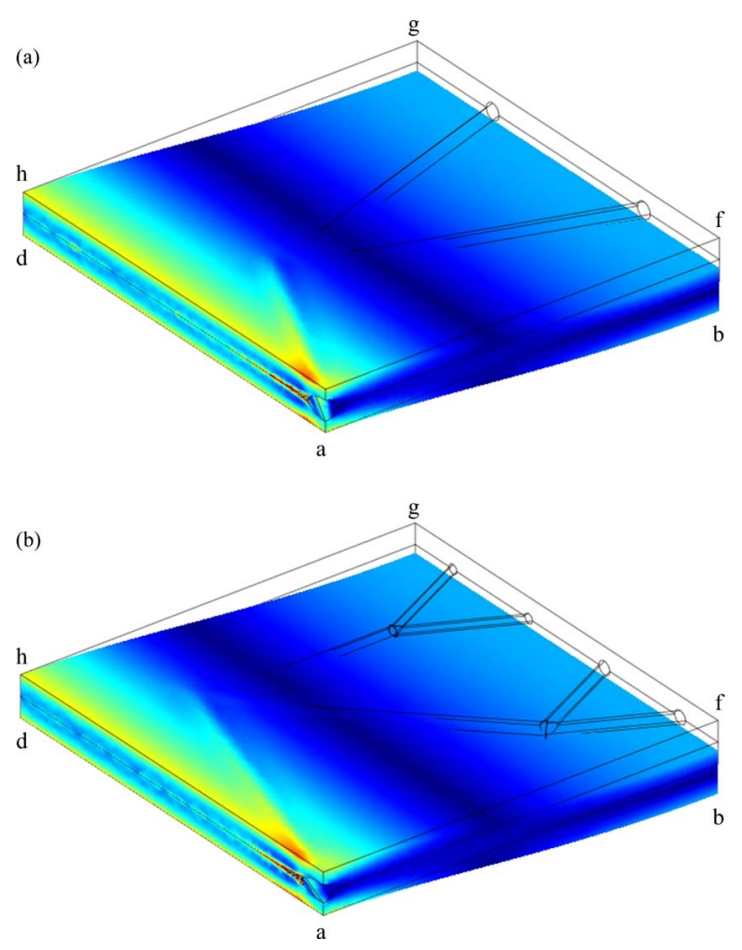

FIG. 10. (Color online) Stress distribution in the optimized configurations when $\phi=0.03$, (a) one bifurcation level, and (b) two bifurcation levels.

$$
\frac{\Delta \mathrm{P}}{\dot{\mathrm{m}}}=\frac{128 \nu \mathrm{d}}{\pi \mathrm{D}_{\mathrm{h}}^{4}},
$$

where $\nu$ is the kinematic viscosity, $\mathrm{d}$ is the channel length, $\dot{\mathrm{m}}$ is the mass flow rate, and $D_{h}$ is the hydraulic diameter. Equation (41) was nondimensionalized by defining the dimensionless variables

$$
\begin{aligned}
& \Delta \widetilde{\mathrm{P}}=\Delta \mathrm{P} /\left(128 \pi \nu \dot{\mathrm{m}}_{\text {total }} / 4 \times 10^{-6} \times \mathrm{L}^{3}\right), \\
& \widetilde{\mathrm{d}}=\mathrm{d} / \mathrm{L}, \\
& \tilde{\dot{\mathrm{m}}}=\dot{\mathrm{m}} / \dot{\mathrm{m}}_{\text {total }},
\end{aligned}
$$

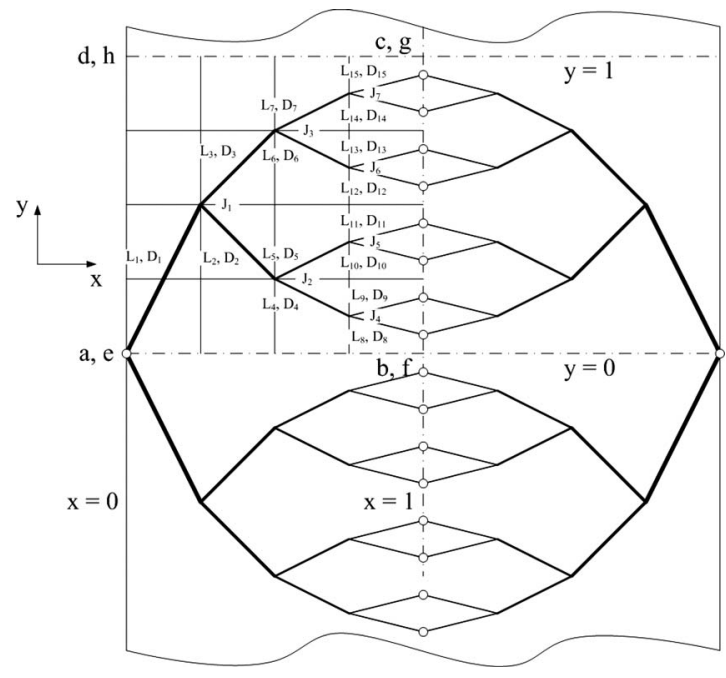

FIG. 11. Flow structure with three levels of bifurcation. 


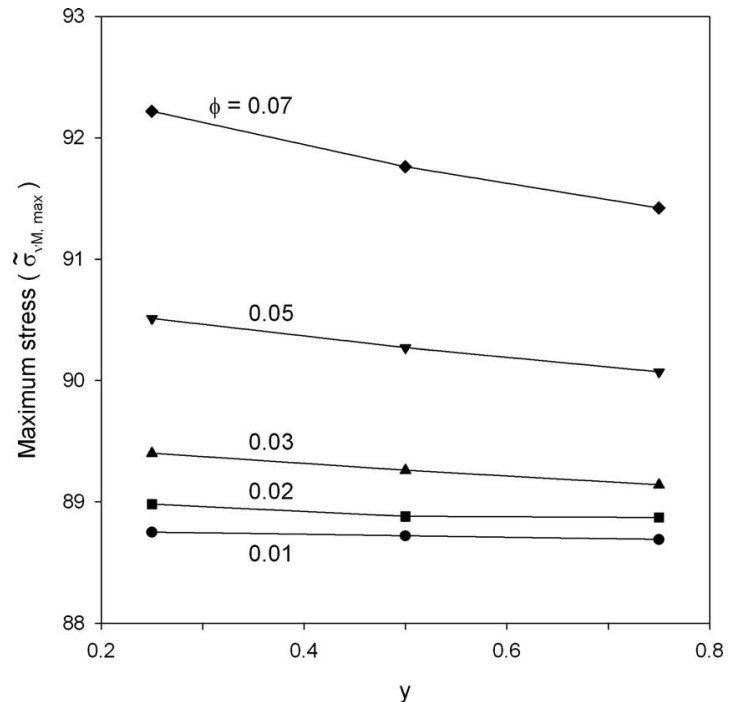

FIG. 12. The effect of the position $y$ of junction $\mathrm{J}_{1}$ on the maximum stresses in the design of Fig. 11.

$$
\tilde{\mathrm{D}}_{\mathrm{h}}=\mathrm{D}_{\mathrm{h}} /\left(\sqrt{4 \times 10^{-3} / \pi} \times \mathrm{L}\right),
$$

where $\dot{\mathrm{m}}_{\text {total }}$ is the total mass flow rate, which is specified. Equation (41) becomes

$$
\frac{\Delta \widetilde{\mathrm{P}}}{\tilde{\mathrm{m}}}=\frac{\tilde{\mathrm{d}}}{\widetilde{\mathrm{D}}_{\mathrm{h}}^{4}},
$$

and the global flow resistance $\Delta \widetilde{\mathrm{P}} / \widetilde{\dot{\mathrm{m}}}$ is plotted in Fig. 14. We see that the design with one bifurcation level has the lowest global flow resistance. The advantages of using free-shaped channels diminish as the porosity increases, however, trees offer lower global flow resistances than the single channel configuration.

\section{CONCLUSIONS}

In this paper, we proposed a design-generation opportunity for structural members with multiple functions such as

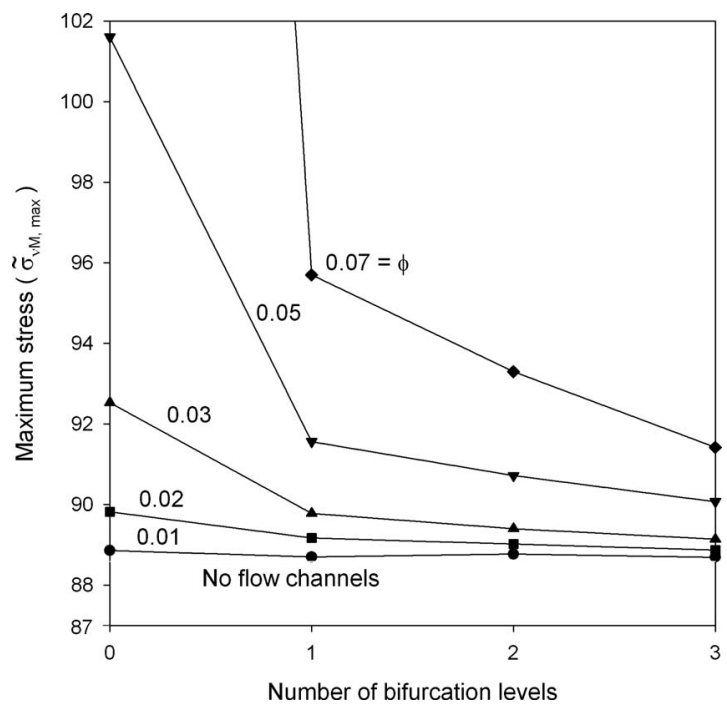

FIG. 13. Minimized maximum stresses of zero-, one-, two-, and threebifurcation flow structures.

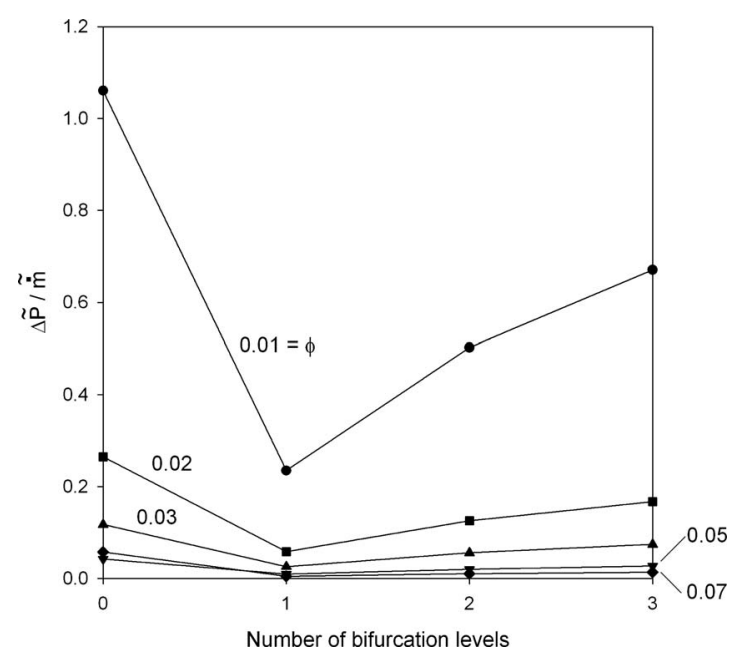

FIG. 14. The global flow resistances of the configurations presented in Fig. 13.

volumetrically distributed cooling, self-healing and variable properties (e.g., effective thermal conductivity). The design concept is to endow the structural member with an embedded vasculature consisting of dentritic channels with multiple length scales and levels of bifurcation. The evolutionary search for such designs is driven by two objectives simultaneously: greater global mechanical resistance and reduced global fluid-flow resistance. This two-objective direction represents a departure from the current state of vascular design, where flow architectures are generated usually by pursuing a single global flow resistance. ${ }^{2}$

We illustrated the generation of vascular designs for strength and fluid flow for several classes of vasculatures, from the simplest to the more complex: parallel channels (Fig. 1), trees with one bifurcation level (Fig. 6), two bifurcation levels (Fig. 8), and three bifurcation levels (Fig. 11). The flow regime in every channel is laminar and fully developed. In each case, we found that it is possible to select not only the channel dimensions but also their positions such that the entire structure offers more strength and less flow resistance when the total volume (or weight) and the total channel volume are fixed. The graphic summary of these results (Fig. 13) shows that the minimized peak stress is smaller when the channel volume $(\phi)$ is smaller and when the vasculature is more complex, i.e., with more levels of bifurcation. Diminishing returns are reached in both direction, decreasing $\phi$, and increasing the complexity. For example, when $\phi=0.02$, the minimized peak stress of a design with one bifurcation level is comparable with (i.e., only $0.2 \%$ greater than) the peak stress in the optimized vascular design with two levels of bifurcation.

\section{ACKNOWLEDGMENTS}

This work was supported by a grant from the U.S. Air Force Office of Scientific Research. 


\section{Nomenclature}

B distance between two parallel channels, $m$

b channel width, $m$

$\mathrm{D}_{\mathrm{h}}$ hydraulic diameter, m, Eqs. (3) and (41)

d channel length, m, Eq. (41)

E Young's modulus, N, Eq. (21)

F force, $\mathrm{N}$

$\mathrm{H}$ wall thickness, $\mathrm{m}$

$\mathrm{h}$ channel height, $\mathrm{m}$

$\mathrm{L}$ side of square slab, $\mathrm{m}$

$\dot{\mathrm{m}}$ mass flow rate, $\mathrm{kg} \mathrm{s}^{-1}$, Eq. (41)

Re Reynolds number, Eq. (7)

$u, v, w$ displacement, $\mathrm{m}$

$\mathrm{W}$ stiffness, $\mathrm{m}^{3}$, Eq. (1)

$\mathrm{V}$ volume, $\mathrm{m}^{3}$

$x, y, z$ Cartesian coordinates, $\mathrm{m}$

\section{Greek symbols}

$\Delta \mathrm{P}$ flow resistance, $\mathrm{N} \mathrm{m}^{-2}$, Eqs. (3) and (41)

$\varepsilon$ normal strain, Eq. (22)

$\phi$ porosity

$\gamma$ shear strain, Eq. (23)

$\nu$ kinematic viscosity, $\mathrm{m}^{2} \mathrm{~s}^{-1}$, Eqs. (7) and (41);

Poisson's ratio, Eq. (21)

$\rho$ density, $\mathrm{kg} / \mathrm{m}^{3}$, Eq. (3)

$\sigma$ normal stress, N, Eqs. (18)-(20)

$\sigma_{\mathrm{vM}}$ von Mises stress, Eq. (32)

$\tau$ shear stress, N, Eqs. (18)-(20)

\section{Superscripts}

() dimensionless, Eqs. (26)-(32)
${ }^{1}$ A. Bejan, Advanced Engineering Thermodynamics, 2nd ed. (Wiley, New York, 1997).

${ }^{2}$ A. Bejan and S. Lorente, Design with Constructal Theory (Wiley, Hoboken, 2008).

${ }^{3}$ C. Dry, Compos. Struct. 35, 263 (1996).

${ }^{4}$ K.-M. Wang, S. Lorente, and A. Bejan, J. Phys. D: Appl. Phys. 39, 3086 (2006).

${ }^{5}$ A. Bejan, S. Lorente and K.-M. Wang, J. Appl. Phys. 100, 033528 (2006).

${ }^{6}$ A. D. Jefferson and T. Bennett, Int. J. Numer. Analyt. Meth. Geomech. 31, 133 (2007)

${ }^{7}$ S. Kim, S. Lorente, and A. Bejan, Int. J. Heat Mass Transfer 50, 3498 (2007).

${ }^{8}$ S. Kim, S. Lorente, and A. Bejan, J. Appl. Phys. 100, 063525 (2006).

${ }^{9}$ K.-M. Wang, S. Lorente, and A. Bejan, J. Phys. D: Appl. Phys. 40, 4740 (2007).

${ }^{10}$ D. S. Burton, X. Gao, and L. C. Brinson, Mech. Mater. 38, 525 (2006).

${ }^{11}$ S. Kim, S. Lorente, and A. Bejan, Int. J. Heat Mass Transfer 51, 5877 (2008).

${ }^{12}$ V. Sauvant-Moynot, S. Gonzalez, and J. Kittel, Prog. Org. Coat. 63, 307 (2008).

${ }^{13}$ K.-M. Wang, S. Lorente, and A. Bejan, Int. J. Heat Mass Transfer 52, 1230 (2009).

${ }^{14}$ S. Kim, S. Lorente, A. Bejan, W. Miller and J. Morse, J. Appl. Phys. 103, 123511 (2008).

${ }^{15}$ A. D. Jefferson and T. Bennett, "Model for cementitious composite materials based on micro-mechanical solutions and damage-contact theory," Comput. Struc. (in press).

${ }^{16}$ J. Lee, S. Kim, S. Lorente, and A. Bejan, Int. J. Heat Mass Transfer 51, 2029 (2008).

${ }^{17}$ J. Wang, A. Gunnion, and A. Baker, Composites, Part A 40, 1433 (2009).

${ }^{18}$ D. Y. Wu, S. Meure, and D. Solomon, Prog. Polym. Sci. 33, 479 (2008).

${ }^{19}$ A. Alibeigloo, Compos. Struct. 92, 113 (2010).

${ }^{20}$ J. P. Den Hartog, Strength of Materials (Dover, New York, 1961).

${ }^{21}$ A. Bejan, Convection Heat Transfer, 3rd ed. (Wiley, Hoboken, 2008).

${ }^{22}$ www.comsol.com 